\title{
A CONTRIBUIÇÃO DA INDEXAÇÃO NO PROCESSO DE SELEÇÃO DE PALAVRAS-CHAVE PARA O GOOGLE ADWORDS
}

\author{
THE CONTRIBUTION OF INDEXING IN THE KEYWORD SELECTION \\ PROCESS FOR GOOGLE ADWORDS
}

\author{
Andréa Nunes de Araújo \\ Cibele Araujo Camargo Marques dos Santos
}

\begin{abstract}
Resumo: O projeto consiste no levantamento bibliográfico do Marketing de Busca e da Biblioteconomia e Documentação, no qual o principal objetivo é contribuir através do uso de técnicas de Indexação, como a de especificação e de associação no processo de seleção de palavras-chave, e conduzi-las no Google Adwords. E depois, a partir da coleta dos assuntos mais acessados no Google Trends, no período de quatro meses (jan-abr), e da realização de testes no planejador de palavraschave do Google, apresentar à avaliação dos resultados comparativos entre os dados coletados e os dados da lista. A partir disso, pretende-se responder se o uso das técnicas de indexação podem ser ou não favoráveis nas criações de campanhas aos clientes brasileiros neste mecanismo de busca.
\end{abstract}

Palavras-chave: Google Adwords, Indexação, Link Patrocinado, Mecanismo de Busca.

\begin{abstract}
The project consists of a bibliographical survey of Search Marketing and Librarianship and Documentation, in which the main objective is to contribute through the use of Indexing techniques, such as specification and association in the process of keyword selection, in the Google Adwords. And then, from collecting the most commonly accessed subjects in Google Trends over the four-month period (Jan-Apr), and conducting tests on Google's keyword planner, to publish results evaluating of the comparison between the collect and list data. From this, it is intended to answer if the use of indexing techniques may or may not be favorable in the creation of campaigns to the brazilians clients of this search engine.
\end{abstract}

Keywords: Google Adwords, Indexing, Search Marketing, Sponsored Link.

\section{Introdução}

O processo de seleção de palavra-chave para utilização nos anúncios em links patrocinados no Google, no caso, a ferramenta é o Google Adwords, de forma que quando uma empresa deseja aparecer em destaque neste mecanismo de busca, pode comprar espaço na página principal, o que funciona como um leilão de palavras-chave, aquela disposta a oferecer o maior lance por uma determinada palavra, terá maior relevância no resultado de busca.

Neste cenário de análise, utilizaremos outra ferramenta do pacote Google, oferecida aos seus anunciantes, conhecida como Google Trends - que disponibiliza os assuntos mais acessados na Internet, e impacta na concorrência das palavras-chave, de forma que os assuntos mais interessantes resultarão em palavras-chave de maior custo ao anunciante. 
VI Seminário de Pesquisa em Ciência da Informação do PPGCI 2017

Escola de Comunicações e Artes - Universidade de São Paulo

Dessa maneira, a hipótese a ser levantada por este projeto é se as técnicas (especificação e associação) de indexação da Biblioteconomia poderão contribuir beneficamente nos resultados comparativos, e se poderão ser aplicadas na criação de campanhas no Google Adwords. Geralmente, as técnicas utilizadas para seleção de palavraschave pertencem ao Marketing Digital, que não possuí o foco da Organização e Representação do Conhecimento que é voltado para a recuperação da informação, como acontece na Ciência da Informação, principalmente nos estudos de indexação da Biblioteconomia e Documentação, sendo este processo também necessário e importante para obtenção de bons resultados de pesquisa em ambientes digitais.

O artigo de Tim Bourgeois (2016), diz que o Google ao lado do Facebook são os dois grandes gigantes do marketing digital, e que no primeiro semestre de 2016 receberam $85 \%$ do mercado total de publicidade na internet. Existe a procura de empresas de médio e pequeno porte por anunciar no Google Adwords, devido à possiblidade de delimitar o orçamento de campanha na ferramenta, assim tornando-se mais acessível aos orçamentos mais limitados.

Dessa maneira, busca contribuir através do uso das técnicas de Indexação da Biblioteconomia, como a de especificação e de associação no processo de seleção de palavras-chave, e conduzi-las no Google Adwords, e na parte aplicada desta pesquisa será criada uma lista a partir do assunto mais acessado no Google Trends, lista que estará delimitada ao território brasileiro e no idioma português, que posteriormente poderá apresentar os resultados benéficos a serem aplicados pelos clientes brasileiros em suas campanhas na ferramenta de anúncio do Google. Assim, estão definidos os seguintes objetivos específicos:

- Refletir sobre os reflexos dos algoritmos nos anúncios do Google, para compreender fatores internos do mecanismo no resultado de relevância de palavras-chave, de forma que por tratar de algoritmos, será necessário rever o conceito de representação do conhecimento pela Inteligência Artificial, pois é a disciplina que estuda o conceito para aprimoramento da interação humano-máquina na web;

- Apresentar a definição do Google Adwords e o funcionamento desta ferramenta no Google;

- Discutir a importância da indexação na seleção de palavras-chave no aporte teórico da Biblioteconomia e Documentação, principalmente sobre a indexação automática, devido ao fato de estudarem as técnicas de recuperação da informação na internet;

- Coletar os assuntos mais acessados no Google Trends, selecionar o principal assunto, e a partir deste, desenvolver uma lista de palavras-chave conforme as técnicas de 
VI Seminário de Pesquisa em Ciência da Informação do PPGCI 2017

Escola de Comunicações e Artes - Universidade de São Paulo

especificação e associação da indexação, e depois testá-las no planejador de palavras-chave do Google Adwords, e realizar um comparativo entre ambas.

\section{Quadro Teórico de Referência}

As principais questões a serem abordadas nesta pesquisa, estão relacionadas aos algoritmos do Google e suas influências nos resultados de busca, o funcionamento do Google Adwords, e a teoria de Indexação no processo de seleção de palavras-chave, para isto foram selecionados pesquisadores da área de Marketing Digital e da Biblioteconomia.

Na primeira questão, utilizaremos o artigo de Larry e Sergey (1999), os fundadores do Google, para explicar como funciona o algoritmo da Page Rank. Além, dos autores que discutem sobre este mecanismo de busca, como Davis (2010) empresário de tecnologia, e Vaidhyanathan (2011) estudioso das mídias digitais. Nestas considerações, entendemos que os algoritmos do Google garantem resultados mais precisos e relevantes, e isto também refletirá nas campanhas criadas no Google Adwords, pois estas variações nos resultados de buscas são responsáveis pela variabilidade dos valores monetários de cada palavra-chave pesquisada no buscador.

Em seguida, ainda em Davis (2010), e também em uma das principais pesquisadoras de Marketing Digital no Brasil, Marta Gabriel (2009 e 2010), apresentamos a definição de Google Adwords e seu funcionamento. Tendo em vista, as agências de publicidade e os setores de marketing de uma empresa, que são os responsáveis pela aplicação de técnicas conhecidas como marketing de busca, em que o foco é mais a venda do que a recuperação da informação.

O Google Adwords trata da venda de publicidade para empresas interessadas em anunciar no buscador na procura de maior visibilidade no meio virtual. $\mathrm{O}$ diferencial da propaganda no Adwords é a personalização do anúncio. Essa é chamada de campanha, que contém informações de onde e quando os anúncios serão apresentados ao público alvo específico. Se a campanha não estiver funcionando podemos alterá-la para melhor adequar ao público ou até mesmo desativá-la.

A partir disso, partimos aos estudos de Ciência da Informação e da Biblioteconomia, em particular da área de Organização e Representação do Conhecimento, que HjØrland (2008) fala ser responsável pela organização dos fluxos de informação, em torno de atividades como descrição do documento, indexação e classificação em unidades de informação e ambientes digitais, que incluem os métodos automatizados de recuperação da informação. 
VI Seminário de Pesquisa em Ciência da Informação do PPGCI 2017

Escola de Comunicações e Artes - Universidade de São Paulo

Com os autores clássicos da Biblioteconomia Lancaster, Borko, Fungman, Cleveland e Cleveland faremos uma discussão sobre a teoria da indexação, a sua definição, sua função e aplicabilidade na internet. Os estudos nos dizem que os índices são produtos da indexação, e surgiram da necessidade humana, como forma de organização da memória com a finalidade de sua posterior recuperação. Portanto, os índices existem desde a antiguidade para facilitar a vida dos indivíduos, pois identificam objetos e informações para algum tipo de uso.

Devido a uma grande quantidade de informação, tornou-se impossível organizá-la por mãos humanas, então foi necessário o uso de robots para localizar e identificar todo o material, para depois ter condições de recuperá-los através de ferramentas como os buscadores da web. Entretanto, Fungman (1993), fala que deve existir um trabalho conjunto entre processos mecanizados e processos manuais de indexação, pois a lógica algorítmica nem sempre acompanhará toda amplitude da linguagem natural, principalmente na tradução de termos quando solicitadas nas buscas em sistemas de informação.

Nesta corrente, se anterior à criação de campanha no Google Adwords, estiverem estabelecidas nessa regra, e concomitantemente estes termos associados estiverem representados no site rastreados pelas aranhas/rastreadores do Google, a alta revocação e precisão ao final da campanha estarão garantidas.

\section{Metodologia, métodos e procedimentos}

A pesquisa tem caráter exploratório, em que o método vai do dedutivo ao indutivo. As fontes utilizadas para a realização deste estudo são empíricas e documentais, no primeiro caso trazendo a experiência da arte de indexar palavras-chave e, no segundo caso, trazendo bibliografia da área de Biblioteconomia e Documentação. A seguir são relatadas as três etapas necessárias para realização desta investigação e os instrumentos para sua realização:

Levantamento bibliográfico sobre o funcionamento de algoritmos de busca do Google, estratégias do marketing digital ao Google Adwords, e da indexação na Biblioteconomia.

Coleta de dados, amostra dos assuntos mais acessado no Google Trends, no caso, a seleção do principal assunto será o futebol, para elaboração de uma lista com palavras-chave, utilizando as técnicas de indexação da Biblioteconomia mais adequadas ao ambiente web.

Teste da lista de termo no planejador de palavras-chave do Google, com avaliação dos resultados a partir do comparativo entre os dados coletados e os dados da lista, com intuito de responder a hipótese desta investigação. 
VI Seminário de Pesquisa em Ciência da Informação do PPGCI 2017

Escola de Comunicações e Artes - Universidade de São Paulo

\section{Conclusão}

A internet está a cada dia mais presente na vida das pessoas, o que representa um aumento no uso de dispositivos móveis, lojas on-line e representações virtuais de serviços utilizados no mundo para além dela. O Google é um ícone daquilo que as pessoas mais acessam quando se fala sobre o assunto, o que atrai atenção de empresas como meio de anunciar os seus serviços e produtos.

A partir disso, a investigação pretende responder se o uso das técnicas de indexação podem ser ou não favoráveis nas criações de campanhas aos clientes deste mecanismo de busca. Conclui-se, portanto, oferecer uma contribuição da área de Biblioteconomia e Documentação no processo de seleção de palavras-chave na criação de anúncios pagos no Google.

\section{Referências}

BORKO, Harold; BERNIER, Charles L. Indexing Concepts and Methods. New York: Academic Press, 1978.

BRIN, Sergey; PAGE, Lawrence. The anatomy of a Large-Scale Hypertextual Web Search Engine. In: Seventh International World-Wide Web Conference (WWW 1998), Stanford Infolab Publication Server, Brisbane, apr. 1998. Disponível em: $<$ http://ilpubs.stanford.edu:8090/361/>. Acesso 28 mar. 2016.

CLEVELAND, Donald B; CLEVELAND, Ana D. Introduction to indexing and abstracting. 3 ed. Colorado: A division of Greenwood Publishing Group, 2001.

DAVIS, Harold. Ferramentas de publicidade do Google: como ganhar dinheiro com o Adsense, o Adwords e as APIs do Google. São Paulo: Novatec Editora, 2010.

FUNGMAN, Robert. Subject analysis and indexing: theoretical foundation na pratice advice. Frankfurt: Indeks Verlag, 1993.

GABRIEL, Marta. Marketing na Era Digital: conceitos, plataformas e estratégias. São Paulo: Novatec Editora, 2010.

GABRIEL, Marta. SEM e SEO: dominando o marketing de busca. São Paulo: Novatec Editora, 2009.

HJØRLAND, B. What is Knowledge Organization (KO)? Knowledge Organization, v.35, n.2/3, p. 86-101, 2008.

LANCASTER, F. W. Indexação e resumos: teoria e prática. 2ed. Brasília: Brinquet de Lemos, 2004.

TIM, Bourgeois. Understanding the Google Marketing Stack. Econtent, Wilton, v. 39, n. 6, 2016. Disponível em: < https://search.proquest.com/docview>. Acesso em: 05 jun. 2017.

VAIDHYANATHAN, S. A Googlelização de tudo. São Paulo: Cultrix, Kindle Edition, 2011. 
VI Seminário de Pesquisa em Ciência da Informação do PPGCI 2017

Escola de Comunicações e Artes - Universidade de São Paulo

\section{Informações da Autora:}

Andréa Nunes de Araújo

Mestranda do Mestrado Profissional em Gestão da Informação - ECA-USP/SP-Brasil

Bibliotecária

Currículo Lattes: http://lattes.cnpq.br/1254789155822465

Email: andreanunes@,usp.br

\section{Cibele Araujo Camargo Marques dos Santos}

Doutora em Ciência da Informação pela Escola de Comunicações e Artes da Universidade de São Paulo

Professora Doutora da Escola de Comunicações e Artes da Universidade de São Paulo 